\title{
Magnetic Resonance Imaging Image under Low-Rank Matrix Denoising Algorithm in the Diagnosis and Evaluation of Tibial Plateau Fracture Combined with Meniscus Injury
}

\author{
Qimao Fu $\mathbb{D}$, Chuizhi Huang $\mathbb{D}$, Yan Chen $\mathbb{D}$, Nailong Jia $\mathbb{D}^{D}$, Jinghui Huang $\mathbb{D}$, \\ and Changkun Lin \\ Department of Radiology, The Second Affiliated Hospital of Hainan Medical University, Haikou 570311, Hainan, China \\ Correspondence should be addressed to Changkun Lin; linchangkun6586@hainmc.edu.cn
}

Received 11 September 2021; Revised 5 November 2021; Accepted 8 November 2021; Published 24 November 2021

Academic Editor: M Pallikonda Rajasekaran

Copyright (c) 2021 Qimao Fu et al. This is an open access article distributed under the Creative Commons Attribution License, which permits unrestricted use, distribution, and reproduction in any medium, provided the original work is properly cited.

\begin{abstract}
This study was carried out to explore the diagnostic effect of magnetic resonance imaging (MRI) based on the low-rank matrix (LRM) denoising algorithm under gradient sparse prior for the tibial plateau fracture (TPF) combined with meniscus injury (TPF $+\mathrm{MI})$. In this study, the prior information of the noise-free MRI image block was combined with the self-phase prior, the gradient prior of MRI was introduced to eliminate the ringing effect, and a new MRI image denoising algorithm was constructed, which was compared with the anisotropic diffusion fusion (ADF) algorithm. After that, the LRM denoising algorithm based on gradient sparse prior was applied to the diagnosis of 112 patients with TPF + MI admitted to hospital, and the results were compared with those of the undenoised MRI image. Then, the incidence of patients with all kinds of different meniscus injury parting was observed. A total of 66 cases (58.93\%) of meniscus tears (MT) were found, including 56 cases $(50.00 \%)$ of lateral meniscus (LM), 10 cases $(8.93 \%)$ of medial meniscus (MM), 16 cases (14.29\%) of meniscus contusion (MC), and 18 cases (16.07\%) of meniscus degenerative injury (MDI). The incidences of MI in Schatzker subtypes were $0 \%, 53.33 \%$ (24/45), $41.67 \%$ (5/12), 76.47\% (13/17), 78.94\% (15/19), and 23.53\% (4/17), showing no statistically significant difference $(P>0.05)$, but the incidence of MT was $54.46 \%$ (61/112), which was greatly higher than that of MC $(15.18 \%(17 / 112))$, and the difference was statistically obvious $(P<0.05)$. The diagnostic specificity $(93.83 \%)$ and accuracy $(95.33 \%)$ of denoised MRI images were dramatically higher than those of undenoised MRI images, which were $78.34 \%$ and $71.23 \%$, respectively, showing statistically observable differences $(P<0.05)$. In short, the algorithm in this study showed better denoising performance with the most retained image information. In addition, denoising MRI images based on the algorithm constructed in this study can improve the diagnostic accuracy of MI.
\end{abstract}

\section{Introduction}

Tibial plateau fracture (TPF) is an important and critical joint fracture or complex fracture of tissue destruction. At present, due to the popularity of electric vehicles and automobiles and the increasing aging population, the number of patients with TPF has generally increased [1]. Among them, the meniscus is the buffer structure between the femoral condyle and the tibial plateau articular surface. Its damage is the most common. Research results show that meniscus injury (MI) is one of the important factors that cause arthritis. The incidence rate is as high as $49 \%$, which can cause physical damage and psychological double torture to patients $[2,3]$. Therefore, improving the diagnostic accuracy of tibial plateau fracture combined with meniscus injury $(\mathrm{TPF}+\mathrm{MI})$ is the top priority of joint medical imaging. Magnetic resonance imaging (MRI) examination has become a common and important means of medical diagnosis. However, MRI examination is easily affected by noise in imaging, so that most of the MRI images obtained have different intensities of noise, which causes abnormally blurred medical image tissue edges, greatly affecting the accurate diagnosis of the disease $[4,5]$. In recent years, denoising methods based on filtering, transform domain, and statistical learning have sprung up, and many researchers are eager to learn, especially methods based on 
prior knowledge of image blocks. Farkas et al. [6] proved that the combination of the Gaussian mixture model (GMM) and maximum A posteriori algorithm based on expectation maximization (MAP-EM) can solve the inversion in image processing.

With the development of artificial intelligence (AI) and information, many researchers apply them to the denoising of medical images, and using low-rank matrix (LRM) to denoise medical images has become a new hot spot. Gonçalves et al. [7] added the $F$ norm and the total variation regular term to enhance the stability of the image solution and then used singular value decomposition (SVD) to solve it to improve the denoising effect of the LRM denoising algorithm. Hassan et al. [8] proposed a completely convolutional encoding and decoding network model for the problems in image denoising and super-resolution reconstruction. But its shortcomings cannot be ignored. It requires a lot of image data to train the parameters of the network, and the acquisition of medical image data is also a big challenge $[9,10]$. However, medical images based on AI denoising are rarely reported on the diagnosis of diseases, and the results are mixed. In this study, the prior information of the noise-free MRI image block was combined with the self-phase prior, the gradient prior of MRI was introduced to eliminate the ringing effect, and a new MRI image denoising algorithm was constructed, which was compared with the anisotropic diffusion fusion (ADF) algorithm. After that, the LRM denoising algorithm based on gradient sparse prior was applied to the diagnosis of 112 patients with $\mathrm{TPF}+\mathrm{MI}$ admitted to hospital, and the results were compared with those of the undenoised MRI image, hoping to provide some theoretical references for the study of $\mathrm{TPF}+\mathrm{MI}$ diagnosis based on MRI images under AI algorithms.

\section{Materials and Methods}

2.1. Research Objects. In this study, $112 \mathrm{TPF}$ patients who were admitted to the hospital from February 14, 2019, to April 23, 2020, were selected as the research subjects. There were 68 males and 44 females, aged $24-78$ years (with an average age of $46.30 \pm 11.49$ years). There were 6 cases of crushing injuries, 58 cases of traffic injuries, 12 cases of drop injuries, and 36 cases of fall injuries. All patients underwent MRI examinations for the diagnosis of their injuries. The study had been approved by the Medical Ethics Committee of the hospital, and the patients and their families had understood the situation of the study and signed the informed consent forms.

The inclusion criteria were defined as follows: patients with TPF and normal lower extremity activities before injury, patients aged 24-78 years, patients without a history of ipsilateral knee fracture before, and patients who agreed to surgery.

The exclusion criteria were defined as follows: patients with severe knee joint disease, patients who did not agree to undergo surgery, patients with systemic diseases, patients with fractures around the knee joint on the same side, and patients who cannot cooperate to sign the informed consent.
2.2. LRM Denoising Algorithm of MRI Based on Gradient Sparse Prior. The denoising of images based on LRM showed excellent performance and had been widely used in the denoising of medical images. In order to reduce the noise of MRI images, the prior information of noise-free MRI image blocks was combined with the self-phase prior, which was mixed with the GMM to guide the clustering of noisy MRI image blocks in this study to enhance the denoising effect. In addition, the gradient prior of the MRI was introduced to eliminate the ringing effect, so as to construct a gradient sparse prior-based MRI LRM denoising algorithm.

An image $A$ was divided into several image blocks, and the collection of which could be expressed as $R A=\left(R_{1} A, \ldots\right.$, $\left.R_{a} A, \ldots, R_{m} A\right)$. These image blocks were divided into $N$ categories, then the probability of any one image block $R_{a} A$ can be expressed by GMM, and its expression was given as follows [11]:

$$
P\left(R_{a} A \mid \lambda\right)=\sum_{n=1}^{N} \omega_{n} P_{n}\left(R_{a} A \mid \mu_{n}, \sum_{n}\right)
$$

In equation ( 1$), \lambda=\left(\mu_{1}, \ldots, \mu_{n}, \sum_{1}, \ldots, \sum_{n}, \omega_{1}, \ldots, \omega_{n}\right)$ referred to the set of all kinds of $\mu, \sum$, and $\omega$ in GMM. The probability density function of the $\mathrm{n}$-th Gaussian class was written as follows:

$$
P_{n}\left(R_{a} A \mid \mu_{n}, \sum_{n}\right)=c \cdot \exp \left[-\frac{\left(R_{a} A-\mu_{n}\right)^{T} \sum_{n}^{-1}\left(R_{a} A-\mu_{n}\right)}{2}\right] \text {. }
$$

The normalization constant in equation (2) was $c$; the negative exponent overcame the defect of the Euclidean distance and described the relationship between $R_{a} A$ and $\mu_{n}$. The Gaussian class to $R_{a} A$ was $B=\left(b_{1}, b_{2}, \ldots\right.$, $\left.b_{m}\right), \quad b_{a} \in\{1,2, \ldots, N\}$; the probability of the $n$-th Gaussian class was $R_{a} A(a=1, \ldots, m)$; and the probability of clustering image blocks into $N$ category was given as follows:

$$
P(R A, B \mid \lambda)=\prod_{a=1}^{m} P\left(R_{a} A, b_{a} \mid \lambda\right)
$$

The following equation could be obtained by taking logarithms on both sides of equation (3):

$$
\begin{aligned}
\log P(R A, B \mid \lambda) & =\sum_{a=1}^{m} \log P\left(R_{a} A, b_{a} \mid \lambda\right) \\
& =\sum_{a=1}^{m} \log P\left(b_{a}\right) P\left(R_{a} A \mid b_{a}\right) .
\end{aligned}
$$

The following equation could be obtained by combining equations (1) and (4):

$$
\sum_{a=1}^{m} \log P\left(b_{a}\right) P\left(R_{a} A \mid b_{a}\right)=\sum_{a=1}^{m} \log \omega_{b_{a}} P_{b_{a}}\left(R_{a} A \mid \mu_{b_{a}}, \sum_{b_{a}}\right) \text {. }
$$

The image block collection of noisy $\mathrm{MRI}^{\mathrm{C}}$ was expressed as $R C=\left(R_{1} C, \ldots, R_{a} C, \ldots, R_{m} C\right)$; based on the $\lambda$ of GMM prior, the image block collection was divided into $N$ 
categories, the matrix of the image blocks of the $n$-th category was $R_{n} C=\left(R_{n_{1}}, \ldots, R_{n_{d(n)}}\right)$, and the number of similar image blocks was $d(n)$; then, the decomposition of the matrix was expressed as follows:

$$
\bar{R}_{n} C=Z_{n}+z_{n} .
$$

In equation (6), $Z_{n}$ referred to the LRM, and $z_{n}$ represented the noise matrix; if the noise was distributed independently, the minimized $E\left(Z_{n}\right)$ is written as follows:

$$
E\left(Z_{n}\right)=v\left\|Z_{n}\right\|_{*}+\frac{\left\|\bar{R}_{n} C-Z_{n}\right\|_{f}^{2}}{\sigma^{2}} \sigma,
$$

where $\sigma$ is the noise standard deviation, $v$ is the normal number, and $\|\cdot\|_{*}$ and $\|\cdot\|_{f}$ are the matrix kernel and the Frobenius norm of the matrix, respectively. We can adopt the norm minimization to optimize the solution, SVD of $R_{n} C$ was decomposed as $U \sum V^{T}$, and the following equation can be obtained:

$$
\bar{Z}_{n}=U S_{O}\left(\sum\right) V^{T}
$$

It was supposed that $S_{O}\left(\sum\right)$ referred to the singular value contraction operator in equation (8). Comprehensive analysis showed that the low-rank decomposition denoising model combining the GMM prior and the MRI image similarity prior was expressed as follows:

$$
\begin{aligned}
\left(\bar{A}, \bar{B}\left\{\bar{Z}_{n}\right\}\right)= & \underset{\bar{A}, \bar{B}\left\{\bar{Z}_{n}\right\}}{\arg \min } \frac{v\|C-A\|_{2}^{2}}{\sigma^{2}}-\log P(R A, B \mid \lambda) \\
& +\sum_{n}^{N} E\left(Z_{n}\right) .
\end{aligned}
$$

Since the different distribution of noise signals in MRI increases the difficulty in denoising, and there was a deviation between the two, the MRI image leveling method was proposed, which can be expressed as follows:

$$
A_{\text {final }}=\left(\bar{A}^{2}-2 \sigma^{2} E\right)^{-1 / 2} \text {. }
$$

In equation (10), $E$ referred to a matrix with the same type of $A$. The alternate minimization was adopted in this study to obtain the estimated class label:

$$
B=\underset{n}{\arg \min } P\left(n \mid R_{a} A\right)=\frac{\omega_{n} P_{n}\left(R_{a} A \mid \mu_{n}, \sum_{n}\right)}{\sum_{n=1}^{N} \omega_{j} P_{j}\left(R_{j} A \mid \mu_{j}, \sum_{j}\right)} .
$$

In the equation above, $n=1,2, \ldots, N ; a=1,2, \ldots, m$. The estimated LRM was expressed as

$$
\bar{Z}_{n}=\underset{\bar{Z}_{n}}{\arg \min } \frac{\left\|\bar{R}_{n} A-Z_{n}\right\|_{f}^{2}}{\sigma^{2}}+\tau\left\|Z_{n}\right\| .
$$

The following equation (13) could be obtained by solving equation (12):

$$
\bar{Z}_{n}=U S_{O}\left(\sum\right) V^{T} .
$$

And, the following equation could be obtained by reconstructing the MRI $A$ :

$$
\bar{A}=\underset{A}{\arg \min } v\|C-A\|_{2}^{2}+\sum_{n=1}^{N}\left\|R_{n} A-Z_{n}\right\|_{f}^{2} .
$$

Equation (14) used the following equation to find the result for the second optimization:

$$
\bar{A}=\frac{\left(v C+\sum_{n}\left(\bar{R}_{n}\right)^{T} \bar{R}_{n}\right)}{\left(v E+\sum_{n}\left(\bar{R}_{n}\right)^{T}-\bar{R}_{n}\right)} .
$$

However, a ringing effect would be generated during the aggregation operation. In order to solve this problem, the gradient prior of MRI was introduced. By finding the horizontal and vertical gradients of a noise-free MRI image, it was found that its gradient was sparse, and the Plath distribution was used to describe this characteristic [12]:

$$
F(X \mid \alpha, \beta)=\frac{1}{2 \beta} \exp \left(-\frac{|X-\alpha|^{\varepsilon}}{\beta}\right),
$$

where $\alpha$ refers to the position parameter and $\beta$ is the scale parameter; equation (16) is the Gaussian distribution if $\varepsilon=2$, and it is the Laplace distribution if $\varepsilon=1 . \alpha=0$; the super Laplacian prior of $A$ based on gradient sparsity was given as follows:

$$
F(A) \infty \exp \left(\left.\dashv \Gamma A\right|_{\varepsilon}\right) .
$$

Thus, the final model was expressed as

$$
\begin{aligned}
\left(\bar{A}, \bar{B}\left\{\bar{Z}_{n}\right\}\right)= & \underset{\bar{A}, \bar{B}\left\{\bar{Z}_{n}\right\}}{\arg \min } \frac{v\|c-A\|_{2}^{2}}{\sigma^{2}}+\eta(-\log P(R A, B \mid \lambda) \\
& \left.+\sum_{n}^{N}\left(E\left(Z_{n}\right)\right)\right)+\varphi|\Gamma A|_{P} .
\end{aligned}
$$

In the above equation, $v, \eta$, and $\varphi$ are all regularization parameters. Then, the algorithm applied in this study was constructed.

The schematic diagram of the constructed algorithm is shown in Figure 1.

2.3. Simulation Experiment. The simulation environment was described as follows: the platform was MATLAB, the operating system was Windows 10 , and the processor was Intel(R) Core (TM) $3 \mathrm{CPU}(70 \mathrm{GHz})$, with a memory of $8 \mathrm{~GB}$. According to experience, the parameter Gaussian category number $N=100 ; v, \eta$, and $\varphi$ were set to $0.18,1$, and 0.75 , respectively; and the image block size was $15 \times 15$. The constructed algorithm was compared with the ADF method to verify the superiority. 


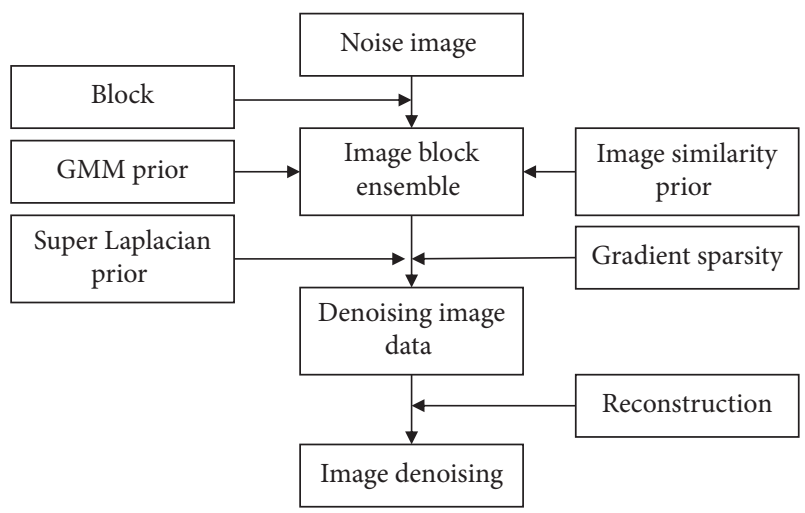

FIGURE 1: Schematic diagram of the constructed algorithm.

The convergences, the peak signal-to-noise ratio (PSNR), and the structural similarity (SSIM) of the two algorithms were compared under the noise intensity of $4,8 \%, 12 \%, 16 \%$, and $22 \%$.

2.4. Imaging Examination of Knee Joint. MRI scanning system (GE7Discovery MR750W) was adopted. During the scan, the patient was required to maintain a supine posture and the knee joints were kept straight. After that, a sandbag was used to fix the calf while the knee joints were naturally rotated at an angle of $20^{\circ} \sim 30^{\circ}$. The patient's knee joints were placed in the center of the MRI examination coil and maintained still. The scan parameters were given as follows: (1) proton density weighted (PDW) (sagittal): time of repetition (TR) was $1400 \mathrm{~ms}$, and time of echo (TE) was $40 \mathrm{~ms}$; (2) selective partial inversion recovery (SPIR) (sagittal): TR was $1148.23 \mathrm{~ms}$, and TE was $7.4 \mathrm{~ms}$; (3) T2-weighted (T2W)-turbo spin-echo (TSE) (coronal): TR and TE were $2763.21 \mathrm{~ms}$ and $60 \mathrm{~ms}$, respectively; and (4) T1-weighted (T1W)-TSE (coronal): TR and TE were $453.56 \mathrm{~ms}$ and $25 \mathrm{~ms}$, respectively. The layer spacing was $4 \mathrm{~mm}$, the interval was $1 \mathrm{~mm}$, and the matrix was $245 \times 245$. During the scan, the sagittal and coronal positions were the main ones, and the center of the scan was the joint space. Two experienced senior physicians in the radiology department were invited to read and evaluate the images.

2.5. Diagnostic Criteria and Surgical Treatment. Kfuri and Schatzker subtype and surgical treatment of TPF were performed based on the MRI results of the patient's knee [13]. The specific classification indexes are as follows: type I fracture was manifested as simple collapsed fractures of the lateral platform; type IV fracture was manifested as medial platform fractures; type $\mathrm{V}$ fracture was featured with bilateral platform fractures; and type VI fracture was combined with a plateau fracture with complete metaphysis. Among them, type IV fracture was mostly local high-energy violent impact injuries and accompanied by knee ligament dislocation, leading to joint injuries such as lateral collateral suture, anterior femoral cruciate ligament, and LM, whereas the local spine fractures in type V and VI fractures were mostly caused by small local bone and high-energy bone injury lesions, causing serious large local bone and soft tissue injuries at the same time.

The patient was required to keep supine, tied with an air tourniquet on the thigh, and then anesthetized and disinfected. The patient had to bend the knee joint at $30^{\circ}$ to install a homeopathic traction quick reducer, so as to check whether the connection was correct. Next, the patient was performed with the homeopathic traction to observe the fracture reduction. If there was no articular surface collapse, it was fixed after reduction. Unilateral platform fractures were fixed with unilateral anatomical bone plates, and bilateral platform fractures or complex platform fractures with metaphyseal fractures were fixed with bilateral anatomical plates. If there was articular surface collapse, a push rod was adopted to press against the collapsed fracture, and then, a double cortical iliac strip was implanted in the bone defect below the joint. If the patient had not recovered the width of the platform after the reduction, a compression bolt can be used to restore the platform width. After the patient's arthroscopy, conservative treatment was adopted for meniscus combined with contusion or partial laceration. For complete tears (longitudinal tears on the side of the joint capsule and barrelhandle tears), they were repaired or removed. Degenerative changes (rough free margins, brush-like changes) should be trimmed; combined cruciate ligament parenchymal fractures or start and end avulsion fractures should be left for secondary reconstruction and plaster fixed. The chief physician was required to perform the TPF surgery and meniscus injury detection and repair surgery [14].

2.6. Observation Indicators. The MI situation was recorded, and the sensitivity, specificity, and accuracy of denoised MRI diagnosis and undenoised MRI diagnosis were calculated according to the equations: sensitivity $=$ true positive/(true positive + false negative); specificity $=$ true negative $/($ true negative + false negative); and accuracy $=($ true positive + true negative)/total.

2.7. Statistical Analysis. The data processing of this study was analyzed by SPSS 22.0 version statistical software. Measurement data were expressed as mean \pm standard deviation $(\bar{x} \pm s)$, and count data were expressed as percentage. Different types of injuries and differences between MRI diagnoses and actual injuries were expressed by $\chi^{2}$ and compared with the square test. The difference was statistically significant at $P<0.05$.

\section{Results}

3.1. Algorithm Simulation Results. Figure 2 shows the convergences of the two algorithms on the simulated brain database (SDB) under $12 \%$ noise conditions. It illustrated that the curve of the ADF algorithm basically remained at $4.044 \times 10^{8}$ at about 25 iterations, while the algorithm constructed basically tended to be flat at about 15 iterations, maintaining at $4.038 \times 10^{8}$. It was said that the convergence 


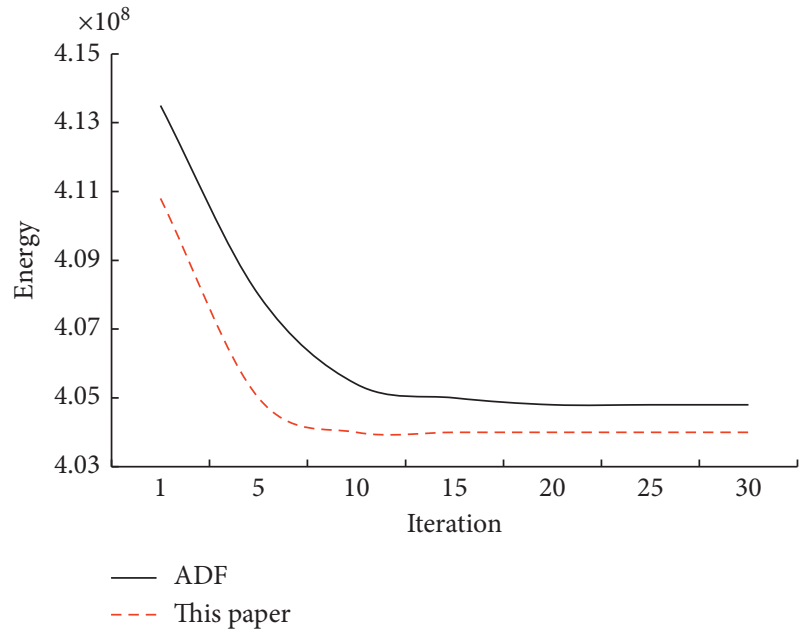

FIGURE 2: Convergences of the two algorithms on the SDB under $12 \%$ noise conditions.

effect of the constructed algorithm was better than that of the ADF algorithm.

Figure 3 shows the PSNR values of the two algorithms under different noise intensities. It disclosed that compared with the algorithm constructed, the PSNR value of the ADF algorithm had increased by $3.267 \sim 7.184 \mathrm{~dB}$, and the PSNR values of the algorithm constructed at $4 \%$ noise $(30.068 \mathrm{~dB})$ and $22 \%$ noise $(15.845 \mathrm{~dB})$ were greatly higher than those of the ADF algorithm, which were $4 \%$ noise $(22.884 \mathrm{~dB})$ and $22 \%$ noise $(9.241 \mathrm{~dB})$, showing statistically obvious differences $(P<0.05)$.

Figure 4 shows the SSIM values of the two algorithms under different noise intensities. Compared with the algorithm in this study, the ADF algorithm showed an increased SSIM value by $0.189 \sim 0.542$, and the SSIM values of the algorithm constructed at $12 \%$ noise $(0.86), 16 \%$ noise (0.809), and $22 \%$ noise $(0.763)$ were much higher in contrast to those of ADF algorithm at $12 \%$ noise $(0.404), 16 \%$ noise $(0.336)$, and $22 \%$ noise $(0.221)$, showing statistically remarkable differences $(P<0.05)$.

3.2. Denoising Results. The denoising results of the two algorithms at $22 \%$ noise intensity are given in Figure 5 . The denoising effect of the ADF algorithm was not obvious, and the proposed algorithm was the closest to the image without noise after denoising, so it had achieved better denoising results compared with the ADF algorithm.

3.3. Schatzker Subtypes and MI Status of All Patients. Figure 6 shows the Schatzker subtypes of all patients. It illustrated that in the Schatzker subtypes, type I accounted for $1.79 \%$ ( 2 cases), type II accounted for $40.18 \%$ (45 cases), type III accounted for $10.71 \%$ (12 cases), type IV accounted for $15.18 \%$ (17 cases), type $\mathrm{V}$ accounted for $16.96 \%$ (19 cases), and type VI accounted for $15.18 \%$ (17 cases).

Figure 7 shows the MI situation of all patients. There were 66 cases (58.93\%) suffered from MT, including 56 cases (50.00\%) of LM and 10 cases (8.93\%) of MM; there were 32

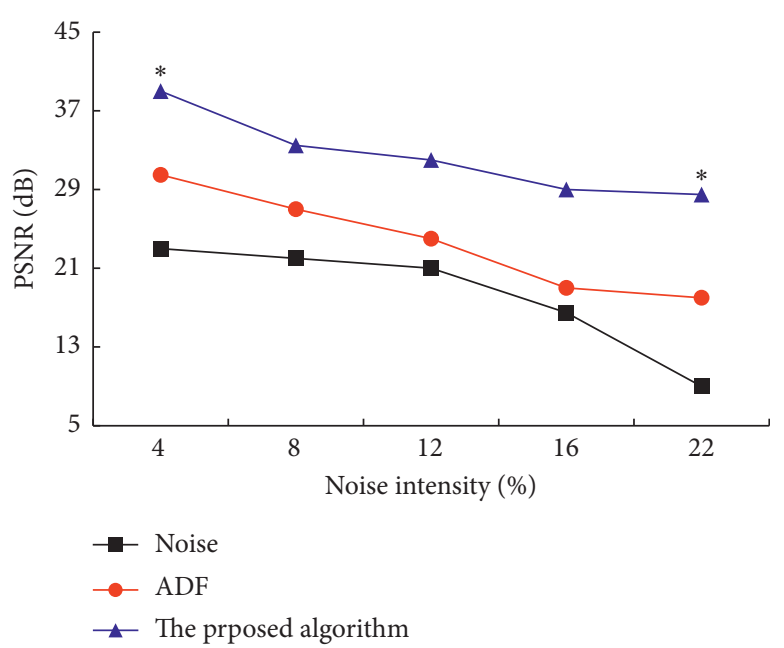

FIGURE 3: PSNR values of the two algorithms under different noise intensities. Note. ${ }^{*}$ means that the difference was statistically visible compared to the ADF algorithm $(P<0.05)$.

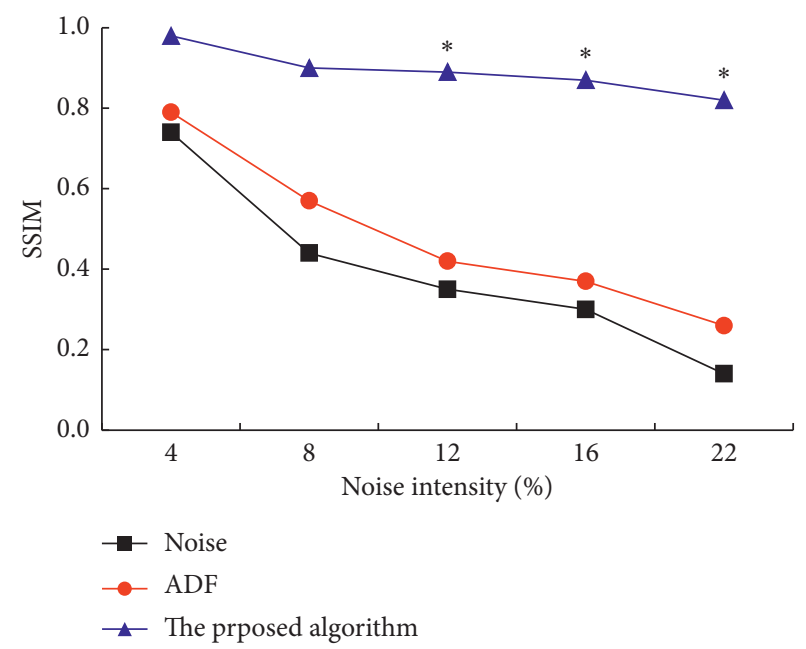

FIGURE 4: SSIM values of the two algorithms under different noise intensities. Note. ${ }^{*}$ means that the difference was statistically visible compared to the ADF algorithm $(P<0.05)$.

cases $(28.57 \%)$ of edge tearing, including 30 cases $(26.79 \%)$ of LM and 2 cases $(1.79 \%)$ of MM; there were 8 cases $(7.14 \%)$ of barrel-handle tearing, with all being LM; there were 8 cases of oblique laceration (7.14\%), including 6 cases of LM (5.36\%) and 2 cases of MM (1.79\%); there were 12 cases of radiation tear $(10.71 \%)$, including 8 cases of LM $(7.14 \%)$ and 4 cases of MM (3.57\%); there were 6 cases of horizontal tearing (5.36\%), including 4 cases of LM (3.57\%) and 2 cases of MM (1.79\%); there were 16 cases of MC (14.29\%), with all being LM; and there were 18 cases of MDI (16.07\%), including 4 cases of LM (3.57\%) and 14 cases of MM (12.50\%).

3.4. MI in Schatzker Subtype Patients. Figure 8 shows the MI of patients with Schatzker subtypes. Among Schatzker type I-VI fractures, the number of MC patients was $0,8,0,6,3$, and 0 , respectively, and that of MT patients was $0,16,5,7,12$, 


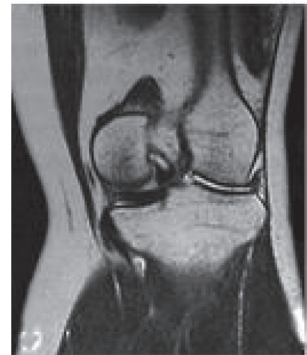

(a)

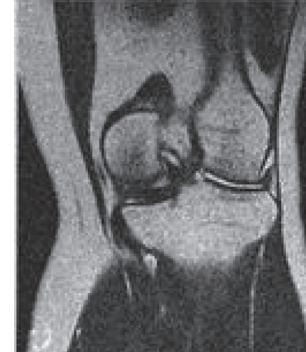

(b)

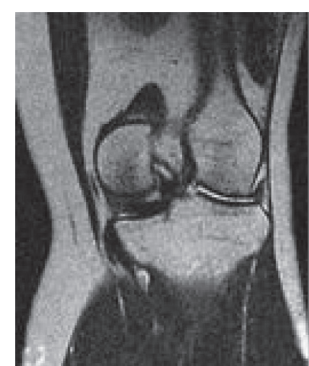

(c)

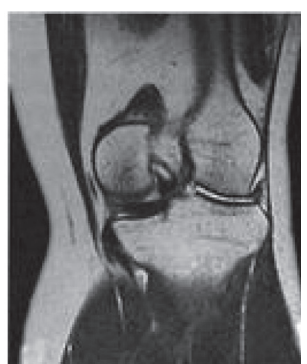

(d)

FIGURE 5: The denoising results of the two algorithms at $22 \%$ noise intensity. (a, c) The clear MRI image and the MRI image denoised with the ADF algorithm, respectively. (b) The MRI image under $22 \%$ noise intensity. (d) The MRI image after denoising with the proposed algorithm.

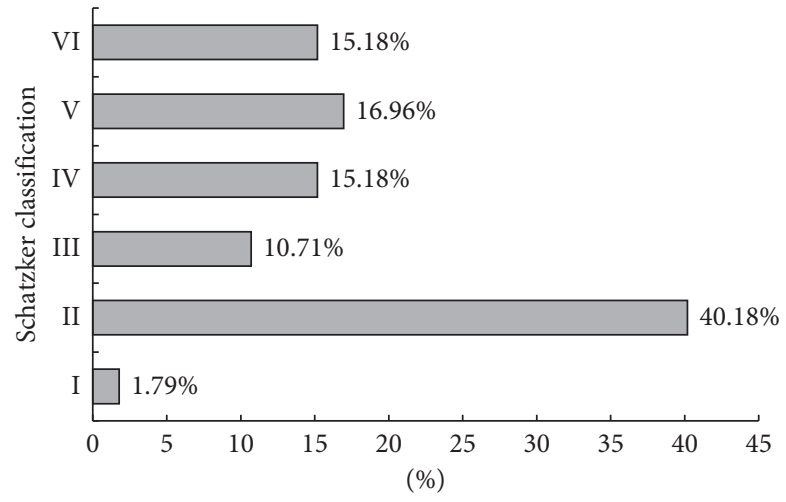

FIGURE 6: The Schatzker subtypes of all patients.

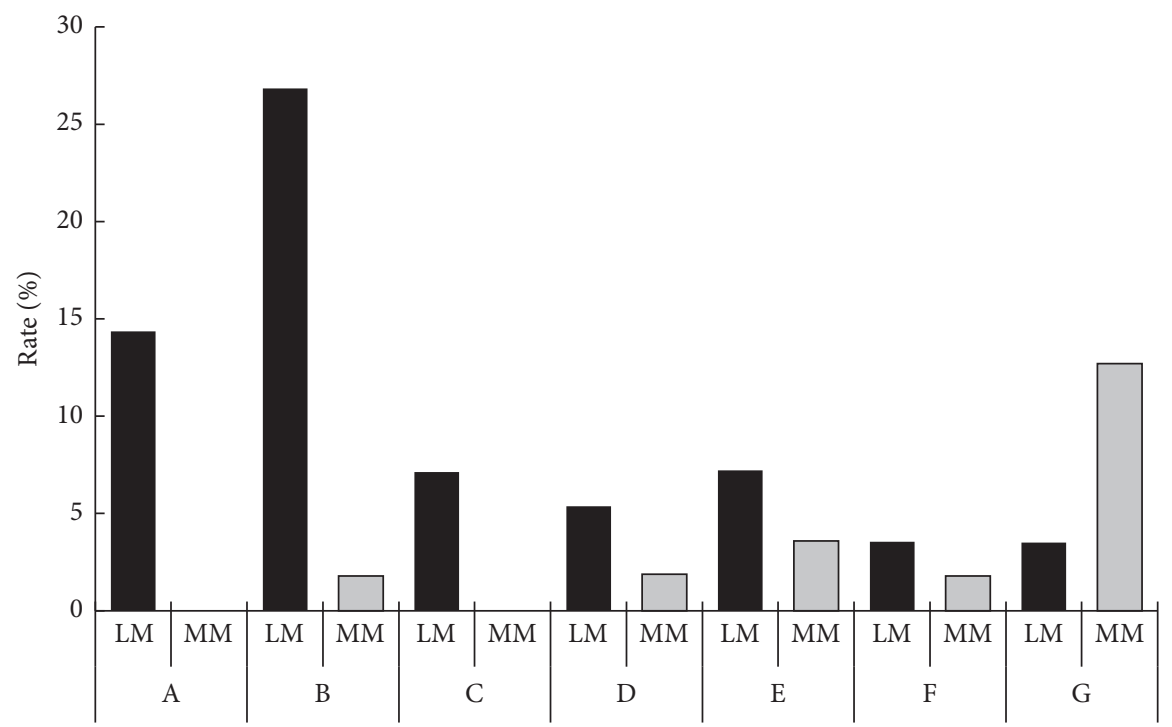

Figure 7: The MI situation of all patients. Note. LM and MM referred to lateral meniscus and medial meniscus, respectively; A G represented contusion, edge tearing, barrel-handle tearing, oblique laceration, radiation tear, horizontal tearing, and meniscus degenerative injury (MDI), respectively.

and 4, respectively. The incidence of MI was $0 \%, 53.33 \%(24 /$ 45), $41.67 \%(5 / 12), 76.47 \%(13 / 17), 78.94 \%(15 / 19)$, and $23.53 \%$ (4/17), respectively. Therefore, the incidence of MI in the Schatzker subtype patients was not statistically significant $(P>0.05)$, but the incidence of MT was $54.46 \%$ $(61 / 112)$, which was much higher in contrast to that of MC. The incidence of $15.18 \%$ (17/112) shows statistically great difference $(P<0.05)$. 


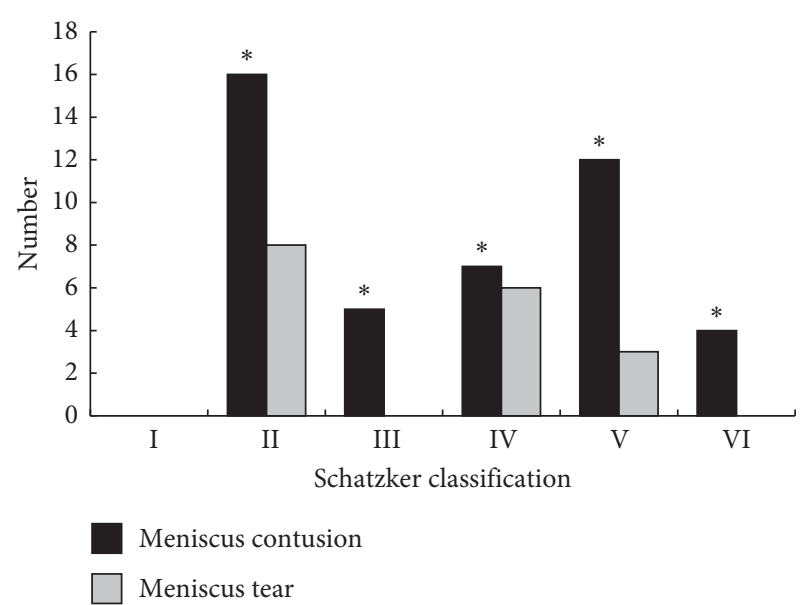

Figure 8: The MI of patients with Schatzker subtypes. Note. * suggests that the incidence of $\mathrm{MI}$ in Schatzker subtype patients was not statistically significant $(P>0.05)$.

3.5. Diagnosis Results of Two Algorithms on MI. The diagnostic sensitivity, specificity, and accuracy of the two methods were compared, and the results are given in Figure 9 . The diagnostic sensitivity of the denoising MRI image was $97.11 \%$, the specificity was $93.83 \%$, and the accuracy was 95.33\%; the diagnostic sensitivity, specificity, and accuracy of MRI images without denoising were $89.31 \%, 78.34 \%$, and $71.23 \%$, respectively. Among them, the diagnostic specificity and accuracy of the denoised MRI images were higher greatly than those of undenoised MRI images, and the differences were statistically visible $(P<0.05)$.

\section{Discussion}

TPF is a complex fracture in which important and critical joints are fractured or tissues are destroyed. There are many types of fractures, among which meniscus injuries are the most common. The meniscus is an important part of the human body, and it acts as a buffer between the femoral condyle and the articular surface of the tibial plateau [15]. A large number of domestic and foreign studies have proved that MI is one of the important factors that cause arthritis, and its incidence is as high as $49 \%$ [16]. Therefore, the diagnosis of TPF + MI is particularly important for the postoperative functional recovery of patients and reducing the incidence of knee joint pain. MRI imaging diagnosis plays an important role in the determination of TPF combined with meniscus injury. Among them, the accuracy and resolution of the image are extremely high, and AIbased MRI image denoising has emerged [17]. In this study, the prior information of the noise-free MRI image block was combined with the self-phase prior, added with GMM to cluster the MRI image block; the gradient prior of MRI was introduced to eliminate the ringing effect, and a new MRI image denoising algorithm was constructed and compared with the ADF algorithm. The results revealed that the convergence effect of the constructed algorithm was better than that of the ADF algorithm. Compared with the ADF algorithm, the PSNR value and SSIM value of the

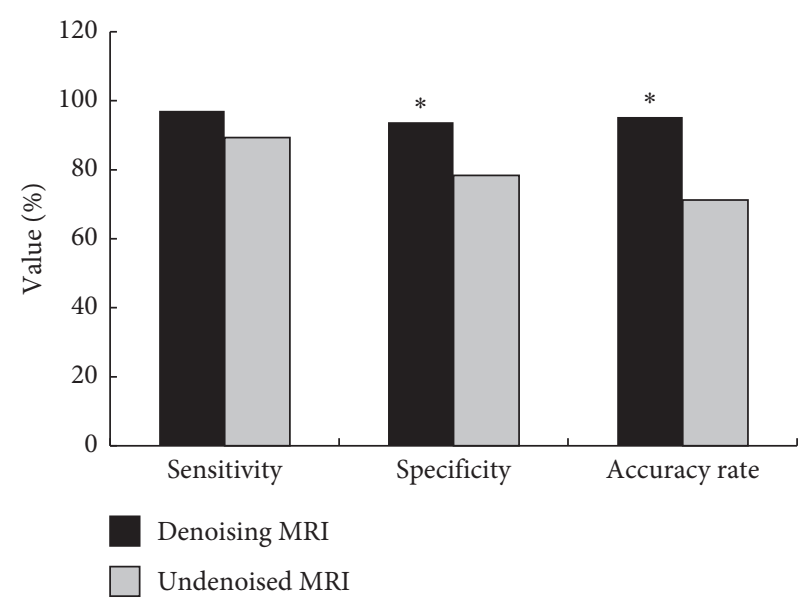

FIGURE 9: The diagnostic sensitivity, specificity, and accuracy of the two methods. Note. ${ }^{*}$ suggests that the difference was statistically obvious in contrast to the diagnostic effect of the undenoised MRI image $(P<0.05)$

constructed new algorithm were improved by 3.267 7.184 dB and 0.189 0.542, respectively. Among them, the PSNR value $(15.845 \mathrm{~dB})$ and SSIM value $(0.763)$ of the algorithm at $22 \%$ noise were significantly higher compared with those of $\mathrm{ADF}$, which were $9.241 \mathrm{~dB}$ and 0.221 , respectively, showing statistically great differences $(P<0.05)$. Compared with the ADF algorithm, the algorithm constructed achieved better denoising results, and the corresponding redundant images hardly contained image detail information, indicating that the denoising results retained the most image information.

After that, the MRI LRM denoising algorithm based on gradient sparse prior was applied to the diagnosis of 112 $\mathrm{TPF}+\mathrm{MI}$ patients admitted to hospital, and the diagnostic sensitivity, specificity, and accuracy of MRI images without denoising were compared. The results showed that, in the Schatzker subtypes, the numbers of patients with types I, II, III, IV, V, and VI were $1.79 \%$ ( 2 cases), $40.18 \%$ (45 cases), $10.71 \%$ (12 cases), $15.18 \%$ (17 cases), $16.96 \%$ (19 cases), and $15.18 \%$ (17 cases), respectively. There were 66 cases (58.93\%) suffered from MT, including 56 cases (50.00\%) of LM and 10 cases $(8.93 \%)$ of MM; there were 32 cases $(28.57 \%)$ of edge tearing, including 30 cases $(26.79 \%)$ of LM and 2 cases (1.79\%) of MM; there were 8 cases $(7.14 \%)$ of barrel-handle tearing, with all being LM; there were 8 cases of oblique laceration $(7.14 \%)$, including 6 cases of LM $(5.36 \%)$ and 2 cases of MM (1.79\%); there were 12 cases of radiation tear (10.71\%), including 8 cases of LM (7.14\%) and 4 cases of MM (3.57\%); there were 6 cases of horizontal tearing $(5.36 \%)$, including 4 cases of LM (3.57\%) and 2 cases of MM (1.79\%); there were 16 cases of MC (14.29\%), with all being LM; and there were 18 cases of MDI (16.07\%), including 4 cases of LM (3.57\%) and 14 cases of MM (12.50\%). Such results were consistent with the findings of Almaawi et al. [18], who selected 103 patients with TPF examined by MRI and found that the incidence of soft tissue injury was as high as $99 \%$, and $91 \%$ of patients had LM injury. The incidences of MI in the Schatzker subtypes were 0\%, 53.33\% (24/45), 41.67\% (5/ 
12), $76.47 \%$ (13/17), $78.94 \%$ (15/19), and $23.53 \%(4 / 17)$, showing statistically remarkable differences $(P>0.05)$; but the incidence of MT was $54.46 \%$ (61/112), which was much higher in contrast to that of MC. The incidence of $15.18 \%$ $(17 / 112)$ shows statistically great difference $(P<0.05)$. Such results were consistent with the descriptions of Polat et al. [19], which revealed that if the lateral platform collapses too much, the risk of LM injury would increase exponentially, indicating that there was a great relationship between the meniscus edge tear and the collapse of the tibial plateau articular surface. The diagnostic specificity (93.83\%) and accuracy (95.33\%) of denoised MRI images were higher obviously than those of undenoised MRI images (78.34\% and $71.23 \%$, respectively), and the differences were statistically observable $(P<0.05)$. It suggested that denoising MRI images based on this algorithm can improve the accuracy of MI diagnosis in patients with TPF + MI. Such outcomes were consistent with the results of Zivanovic et al. [20], who proposed an image denoising algorithm based on LRM, which constrained the sparseness of the noise to ensure the sparsity to restore the image in order to achieve the purpose of denoising.

\section{Conclusion}

In this study, the prior information of the noise-free MRI image block was combined with the self-phase prior, the gradient prior of MRI was introduced to eliminate the ringing effect, and a new MRI image denoising algorithm was constructed, which was compared with the anisotropic diffusion fusion (ADF) algorithm. After that, the LRM denoising algorithm based on gradient sparse prior was applied to the diagnosis of 112 patients with TPF + MI admitted to hospital, and the results were compared with those of the undenoised MRI image. It was found that the MRI LRM denoising algorithm based on gradient sparse prior showed better denoising performance, and the denoising results had the most retained image signals. Denoising MRI images based on the algorithm constructed in this study can improve the accuracy of MI diagnosis. The shortcoming of this study was that the research sample was small and the type was diverse, so that the applicability of the results was limited. Therefore, the sample size can be expanded in the follow-up. In short, the results of this study provided a reliable theoretical reference for the study of TPF + MI diagnosis based on MRI images under the intelligent algorithms.

\section{Data Availability}

The data used to support the findings of this study are available from the corresponding author upon request.

\section{Conflicts of Interest}

The authors declare no conflicts of interest.

\section{Authors' Contributions}

Qimao Fu and Chuizhi Huang contributed equally to this work.

\section{References}

[1] L. Menzdorf, T. Drenck, R. Akoto et al., "Clinical results after surgical treatment of posterolateral tibial plateau fractures ("apple bite fracture") in combination with ACL injuries," European Journal of Trauma and Emergency Surgery, vol. 46, no. 6, pp. 1239-1248, 2020.

[2] Y. Li, J. L. Zhao, Z. H. Lv, and J. H. Li, "Medical image fusion method by deep learning," International Journal of Cognitive Computing in Engineering, vol. 2, pp. 21-29, 2021.

[3] D. Zhao, W. Li, L. Liu, N. Lu, L. Chen, and J. Zhang, "Open tibia plateau fracture with intra-osseous dislocation of the patella and quadriceps tendon rupture: a case report," $B M C$ Surgery, vol. 20, no. 1, p. 114, Apr 15; 2020.

[4] K. Yokoyama, J. Tsuchiya, and U. Tateishi, "Comparison of [18F]fdg PET/CT and MRI for treatment response assessment in multiple myeloma: a meta-analysis," Diagnostics, vol. 11, no. 4, p. 706, 2021.

[5] K. N. Subramanyam, M. Tammanaiah, A. V. Mundargi, R. N. Bhoskar, and P. S. Reddy, "Outcome of complex tibial plateau fractures with Ilizarov external fixation with or without minimal internal fixation," Chinese Journal of Traumatology, vol. 22, no. 3, pp. 166-171, 2019.

[6] B. Farkas, G. Csizmadia, E. Katona, G. E. Tusnády, and T. Hegedüs, "MemBlob database and server for identifying transmembrane regions using cryo-EM maps," Bioinformatics, vol. 36, no. 8, pp. 2595-2598, 2020.

[7] B. Gonçalves, D. Coutinho, J. Exel, B. Travassos, C. Lago, and J. Sampaio, "Extracting spatial-temporal features that describe a team match demands when considering the effects of the quality of opposition in elite football," PLoS One, vol. 14, no. 8, Article ID e0221368, 2019.

[8] A. Hassan, M. Ghafoor, S. A. Tariq, T. Zia, and W. Ahmad, "High efficiency video coding (HEVC)-Based surgical telementoring system using shallow convolutional neural network," Journal of Digital Imaging, vol. 32, no. 6, pp. 1027-1043, 2019.

[9] Y. Shao, M. Hellström, P. D. Mitev, L. Knijff, and C. Zhang, "PiNN: a Python library for building atomic neural networks of molecules and materials," Journal of Chemical Information and Modeling, vol. 60, no. 3, pp. 1184-1193, 2020.

[10] C. Yones, J. Raad, L. A. Bugnon, D. H. Milone, and G. Stegmayer, "High precision in microRNA prediction: a novel genome-wide approach with convolutional deep residual networks," Computers in Biology and Medicine, vol. 134, Article ID 104448, 2021.

[11] Y. Wang, W. Ren, L. Cheng, and J. Zou, “A grey model and mixture Gaussian residual analysis-based position estimator in an indoor environment," Sensors, vol. 20, no. 14, p. 3941, 2020.

[12] O. Hrusak, T. Kalina, J. Wolf et al., "Flash survey on severe acute respiratory syndrome coronavirus- 2 infections in paediatric patients on anticancer treatment," European Journal of Cancer, vol. 132, pp. 11-16, 2020.

[13] M. Kfuri and J. Schatzker, "Revisiting the Schatzker classification of tibial plateau fractures," Injury, vol. 49, no. 12, pp. 2252-2263, 2018.

[14] T. Vendeuvre, O. Monlezun, C. Brandet et al., "Comparative evaluation of minimally invasive 'tibial tuberoplasty' surgical technique versus conventional open surgery for Schatzker IIIII tibial plateau fractures: design of a multicentre, randomised, controlled and blinded trial (TUBERIMPACT study)," BMJ Open, vol. 9, no. 8, Article ID e026962, 2019.

[15] C. Guo, J. Lu, Z. Tian, W. Guo, and A. Darvishan, "Optimization of critical parameters of PEM fuel cell using TLBO-DE based on 
Elman neural network," Energy Conversion and Management, vol. 183, pp. 149-158, 2019.

[16] M. Hu, Y. Zhong, S. Xie, H. Lv, and Z. Lv, "Fuzzy system based medical image processing for brain disease prediction," Frontiers in Neuroscience, vol. 15, Article ID 714318, 2021.

[17] L. Stanke, J. Kubicek, D. Vilimek et al., "Towards to optimal wavelet denoising scheme-A novel spatial and volumetric mapping of wavelet-based biomedical data smoothing," Sensors, vol. 20, no. 18, p. 5301, 2020.

[18] A. Almaawi, W. Awwad, A. Bamugaddam et al., "Prevalence of knee injuries among male college students in Riyadh, Kingdom of Saudi Arabia," Journal of Orthopaedic Surgery and Research, vol. 15, no. 1, p. 126, 2020.

[19] B. Polat, T. Gurpinar, A. E. Polat, and Y. Ozturkmen, "Factors influencing the functional outcomes of tibia plateau fractures after surgical fixation," Nigerian Journal of Clinical Practice, vol. 22, no. 12, pp. 1715-1721, 2019.

[20] M. Zivanovic, M. Niegowski, P. Lecumberri, and M. Gómez, "A low-rank matrix factorization approach for joint harmonic and baseline noise suppression in biopotential signals," Computer Methods and Programs in Biomedicine, vol. 141, pp. 59-71, 2017. 